\section{Wave upon chemical wave}

As indicated in lan Stewart's recent News and

Views article (Nature $\mathbf{3 5 0}$, 557; 1991), coupled oscillators can offer some spectacular sights. The geometric complexity resulting from two coupled chemical-wave systems, pictured on the right, attests further to the fact The spiral waves are formed on a polymer (Nafion) membrane loaded with ferroin and immersed in a mixture of the Belousov-Zhabotinsky reagents. The ferroin catalyses this oscillatory reaction, generating wavefronts that propagate on both sides of the membrane. The two sets of waves can communicate by transport of one reagent through the membrane. On page 132 , K. Showalter et al. show that this inter-

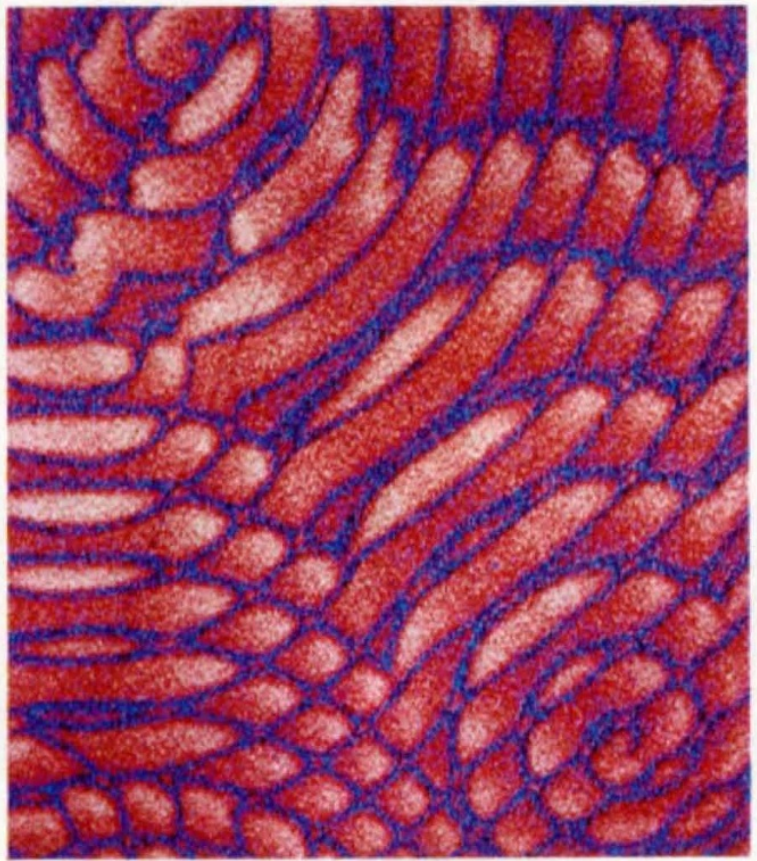

action can lead to behaviour ranging from irregular patterns to entrainment and phase locking. It is tempting to infer that this kind of phase-sensitive communication could occur across cell membranes. The wavefronts are seen here in blue on both sides of the translucent membrane.

recycling, dual recycling can operate over a wide range of bandwidths.

The new work by K. A. Strain and B. J. Meers ${ }^{1}$ demonstrates that dual recycling can improve the broadband low-frequency performance of a particular interferometer configuration which uses optical delay lines to store light in its arms. With this improvement the delay-line interferometer should be as sensitive as a Fabry-Perot interferometer. (Practical constraints on the size of mirrors had previously favoured the more compact Fabry-Perot configuration for detecting gravitational waves at frequencies below a few hundred hertz because the maximum storage time for light in a delay line was too short.) For their demonstration, Strain and Meers built a small-scale broadband recycling interferometer in which the light made a single bounce in each arm. Because the mirrors were not suspended as in a real gravitationalwave detector but were held in rigid mounts, vibrations dominated the displacement noise below $20 \mathrm{kHz}$. Above this frequency, the displacement noise was shot-noise limited at a level determined by the available brightfringe power and the very short storage time of light in the arms.

When the signal recycling mirror was added, a sevenfold increase in signal-tonoise ratio was observed. This increase was slightly better than the expected sixfold improvement, a fact which Strain and Meers attribute to the improved quality of the optical wavefront inside the interferometer owing to dual recycling.

Dual recycling also looks promising to those of us who favour the Fabry-Perot interferometer design. Dual recycling can adapt a broadband recycled interferometer to narrowband operation by adding a single mirror to the output side of the instrument. Adjusting the position of the signal recycling mirror tunes the interferometer to a particular frequency, which might be chosen to correlate with the rotation frequency of a known pulsar. The detector's bandwidth can be varied by adjusting the transmission of the signal recycling mirror. In some cases an intermediate bandwidth may be desirable, trading off some bandwidth for improvements in mode quality within the interferometer.

A very different method for improving interferometer sensitivity in the shot-noise limit is to inject 'squeezed' light into the interferometer at the beam splitter ${ }^{4}$. The presence of squeezed light can alter the stochastic nature of the beam splitting process, reducing quantum fluctuations in the output beam at the cost of increased fluctuations in radiation pressure on the test masses. Although squeezing cannot violate the uncertainty principle, it may allow the quantum limit to be reached with less bright-fringe power. The improvement in precision has been confirmed in a simple interferometer ${ }^{5}$ and the evaluation of squeezing techniques in practical gravitational-wave detectors has begun ${ }^{6}$. For squeezed light to be effective, interferometer losses must be minimized, a situation which also enhances recycling efficiency.

Where will this lead? Gravitational-waves

\section{Fit connection}

Two years after the mapping of the gene which causes a rare form of neonatal epilepsy, a Finnish group reports the localization of the gene for an adult form of the disorder (A.-E. Lehesjoki et al. Proc. natn. Acad. Sci. U.S.A. 88, 3696-3699; 1991). Progressive myoclonus epilepsy of Unverricht-Lindberg type was first described 100 years ago, and is especially common in the Baltic region. Performing linkage analysis with 12 Finnish families, Lehesjoki and colleagues excluded 25 per cent of the genome before tracking down the disease locus to position 22.3 on the long arm of chromosome 21 . The most promising candidate gene which maps to the same region encodes the $\beta$ subunit of the calcium-binding protein S100, expressed mainly in glial cells.

\section{Golden opportunity}

THE discovery in 1984 that certain phospholipids spontaneously arrange themselves into microtubular structures led C. R. Martin and colleagues to see if the use of microporous materials as templates would extend the range of microtubular materials. To the surprise of Martin and C. J. Brumlik (J. Am. chem. Soc. 113, 3174-3175; 1991) these structures are not restricted to organic molecules. Gold can be persuaded to coat the pores of microporous alumina, without blocking them. The alumina can then be etched away to leave tubules with a diameter as little as $0.1 \mu \mathrm{m}$ and a length of up to $2 \mu \mathrm{m}$. One possible application, says Martin, would be to use plugs of gold microtubules, which have a large surface-to-volume ratio, in flowing electrochemical reactors to reduce solutions.

\section{RNA action}

MANY of the processes going on inside living cells are carried out by complex machines made up of both proteins and RNA - perhaps the best known case is the translation of messenger RNA by ribosomes, but other more recently fashionable examples include splicing of RNA and protein export. Inspired by these precedents, L. Young et al. (Science 252, 542-546; 1991)

took another look at the event that creates it all in the first place, transcription of DNA. In eukaryotes, transcription requires, in addition to RNA polymerase itself, a whole array of auxiliary factors, all of which were assumed to be proteins. Not so, it seems. Young and colleagues have found a factor that is essential for transcription by RNA polymerase III in silkworms and that consists, at least in part, of RNA. The number of biological processes that do not require this material shrinks further. 